\title{
Erratum: Influence of the Surface Viscosity on the Breakup of a Surfactant-Laden Drop [Phys. Rev. Lett. 118, 024501 (2017)]
}

\author{
A. Ponce-Torres, J. M. Montanero, M. A. Herrada, E. J. Vega, and J. M. Vega
}

(Q) (Received 12 February 2018; published 2 March 2018)

DOI: 10.1103/PhysRevLett.120.099901

This Letter considered the effect of low surface shear and dilatational viscosities, leading to superficial Ohnesorge numbers $C_{1}^{S}$ and $C_{2}^{S}$ of the order $10^{-3}-10^{-2}$. For this reason, the normal components of the superficial viscous stresses were expected to be much smaller than the surface tension. We forgot mentioning this approximation in the Letter. The balance of normal stresses at the free surface including those components yields

$$
-p+B z+\mathbf{n} \cdot \mathbf{T} \cdot \mathbf{n}=\left[\hat{\sigma}+\left(C_{2}^{S}-C_{1}^{S}\right) \boldsymbol{\nabla}^{S} \cdot \mathbf{v}^{S}\right] \kappa+2 C_{1}^{S}\left[\kappa_{1}\left(\nabla^{S} \mathbf{v}^{S}\right)_{11}+\kappa_{2}\left(\nabla^{S} \mathbf{v}^{S}\right)_{22}\right]
$$

where $\kappa_{1}$ and $\kappa_{2}$ are the curvatures along the meridians and parallels in the inward normal direction $\left(\kappa=\kappa_{1}+\kappa_{2}\right)$, respectively, and $\left(\nabla^{S} \mathbf{v}^{S}\right)_{11}$ and $\left(\nabla^{S} \mathbf{v}^{S}\right)_{22}$ are the diagonal elements of $\boldsymbol{\nabla}^{S} \mathbf{v}^{S}$ along the meridians and the parallels, respectively. If one considers the neglected terms, the variation of the satellite droplet radius and surfactant concentration are around or even lower than $5 \%$, smaller than errors due to the approximations mentioned in the Letter. 